\title{
Reactor neutrons in nuclear astrophysics
}

\author{
René Reifarth $^{1, \text { a }}$, Jan Glorius ${ }^{2}$, Kathrin Göbel ${ }^{1}$, Tanja Heftrich ${ }^{1}$, Michael Jentschel ${ }^{3}$, Beatriz Jurado ${ }^{4}$, Franz Käppeler ${ }^{5}$, \\ Ulli Köster ${ }^{3}$, Christoph Langer ${ }^{1}$, Yuri A. Litvinov ${ }^{2}$, and Mario Weigand ${ }^{1}$ \\ 1 Goethe University Frankfurt am Main, Frankfurt, Germany \\ 2 GSI Helmholtzzentrum für Schwerionenforschung, Darmstadt, Germany \\ 3 Institut Laue-Langevin, Grenoble, France \\ ${ }^{4}$ CENBG, CNRS/IN2 P3, Gradignan, France \\ 5 KIT, Karlsruhe, Germany
}

\begin{abstract}
The huge neutron fluxes offer the possibility to use research reactors to produce isotopes of interest, which can be investigated afterwards. An example is the half-lives of long-lived isotopes like ${ }^{129} \mathrm{I}$.

A direct usage of reactor neutrons in the astrophysical energy regime is only possible, if the corresponding ions are not at rest in the laboratory frame. The combination of an ion storage ring with a reactor and a neutron guide could open the path to direct measurements of neutron-induced cross sections on short-lived radioactive isotopes in the astrophysically interesting energy regime.
\end{abstract}

\section{Introduction}

Almost all of the heavy elements are produced via neutron capture reactions equally shared between $s$ and $r$ process $[1-3]$ and to a very small extent by the $i$ process $[4,5]$. The remaining minor part is produced via photon- and protoninduced reactions during the $p$ process [6], see Fig. 1.

The neutron-induced nucleosynthesis in stars occurs typically at temperatures between $k T=5 \mathrm{keV}$ and $k T=200 \mathrm{keV}$. The neutron-energy distribution in reactors is centered around $25 \mathrm{meV}$. The reaction cross sections at $25 \mathrm{meV}$ are therefore usually not a strong constraint for the reaction cross sections in the $\mathrm{keV}$-regime.

However, the huge neutron fluxes of $10^{12}-10^{15} \mathrm{n} / \mathrm{cm}^{2} / \mathrm{s}$ offer the possibility to use research reactors to produce isotopes of interest, which can be investigated afterwards.

\section{Half-life measurements on long-lived isotopes}

The determination of half lives in the range of $10^{4}-10^{8}$ years is a very challenging task. The reason is that in contrast to shorter half lives, they can only be determined from the ratio of activity and number of nuclei:

$$
t_{1 / 2}^{\text {long }}=\frac{\log 2}{\lambda^{\text {long }}}=\frac{N_{\text {sample }}}{A^{\text {long }}} \log 2
$$

Therefore, the activity must be determined, which is difficult, since usually no $\gamma$-rays are emitted and, even more challenging, the number of atoms has to be determined. The determination of the number of atoms is difficult, because the material has to be freshly produced since it is not naturally abundant on earth. This means, it will only be available in minute amounts. This typically results in huge systematic uncertainties, which is reflected

a e-mail: reifarth@physik.uni-frankfurt.de in contradicting data from different measurements. One possibility to address this problem is to produce a shortlived isotope, which decays to the long-lived isotope. Since the half-life of the short-lived isotope is known, the total amount produced ( $\left.N_{\text {produced }}\right)$ can easily be determined from the short-lived activity. After the decay of the shortlived isotope, the number of atoms in the sample is therefore:

$$
N_{\text {sample }}=N_{\text {produced }}=\frac{t_{1 / 2}^{\text {short }}}{\log 2} A^{\text {short }}
$$

This means, the task of determining the the long halflife is reduced to the measurement of two activities. The activity of the short-lived intermediate isotope just after the irradiation and the activity of the long-lived isotope long after the irradiation, when the short-lived isotope is (almost) completely decayed:

$$
t_{1 / 2}^{\text {long }}=\frac{A^{\text {short }}}{A^{\text {long }}} t_{1 / 2}^{\text {short }}
$$

\subsection{The long-lived decay of ${ }^{129}$ |}

An illustrative example is ${ }^{129} \mathrm{I}$ with a $\beta^{-}$-decay of about $15 \mathrm{Ma}$, Fig. 2. The exact knowledge of the half-life time of ${ }^{129} \mathrm{I}$ is important for several fields. Since ${ }^{129} \mathrm{I}$ is a long-lived fission product, its half-life is of interest for the estimation of the long-term activity of burnt reactor fuel material. The slow decay can be used in cosmology to determine the age of the solar system [7].

Using reactors, it is possible to irradiate ${ }^{128} \mathrm{Te}$, which can capture a neutron. Afterwards one can measure the activity ratio of ${ }^{129} \mathrm{Te}$ to ${ }^{129} \mathrm{I}$, which results in an independent determination of the half-life of ${ }^{129} \mathrm{I}$. If the epithermal neutron flux is sufficiently high, ${ }^{132} \mathrm{Xe}$ could be irradiated and the $(\mathrm{n}, \alpha)$-reaction leads also to ${ }^{129} \mathrm{Te}$. 


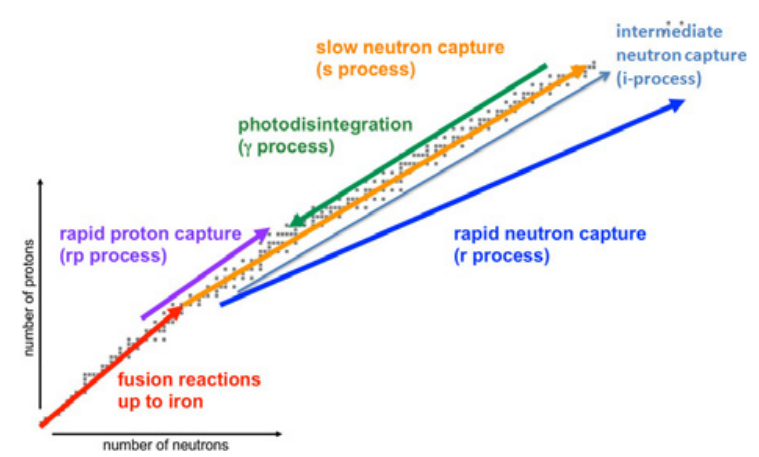

Figure 1. The $s$ and $r$ processes start with the iron peak nuclei as seeds. The s process path follows the nuclear valley of stability until it terminates in the lead-bismuth region. The $\mathrm{r}$ process drives the nuclear matter far to the neutron-rich side of the stability line and upwards until beta-delayed fission and neutron-induced fission occur and recycle the material back to smaller mass numbers. The reaction path of the $i$ process lays in between, since the corresponding intermediate neutron densities are higher than during the $s$ process, but still much smaller than during the $r$ process. Only a few isotopes on the proton-rich side of the valley of stability get significant contributions from the different models of the $p$ process.

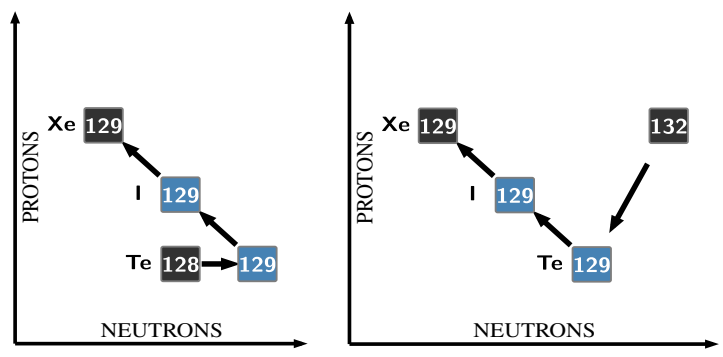

Figure 2. Production of ${ }^{129} \mathrm{I}$ via ${ }^{128} \mathrm{Te}\left(\mathrm{n}, \gamma\right.$ (left) or via ${ }^{132} \mathrm{Xe}(\mathrm{n}, \alpha)$ (right).

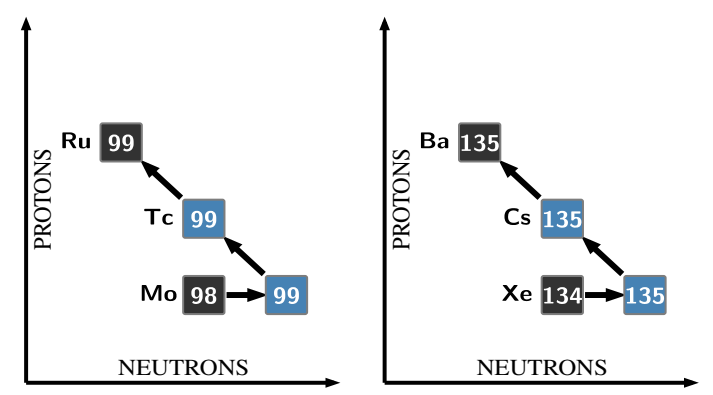

Figure 3. Production of ${ }^{99} \mathrm{Tc}$ via neutron capture on ${ }^{98} \mathrm{Mo}$ (left) and production of ${ }^{135} \mathrm{Cs}$ via neutron capture on ${ }^{134} \mathrm{Cs}$ (right).

\subsection{The long-lived decays of ${ }^{99} \mathrm{Tc}$ and ${ }^{135} \mathrm{Cs}$}

Other examples are ${ }^{99} \mathrm{Tc}$ and ${ }^{135} \mathrm{Cs}$, Fig. 3. These isotopes are branch points of the $s$-process path [3] as well as important long-lived fission products [8]. The production in a reactor can be performed by irradiation of ${ }^{98} \mathrm{Mo}$ or ${ }^{134} \mathrm{Xe}$.

\section{Neutron capture cross sections}

Neutron capture cross sections of stable and unstable isotopes are important for neutron-induced nucleosynthesis [3] as well as for technological applications [8]. As already discussed, the traditional method of determining

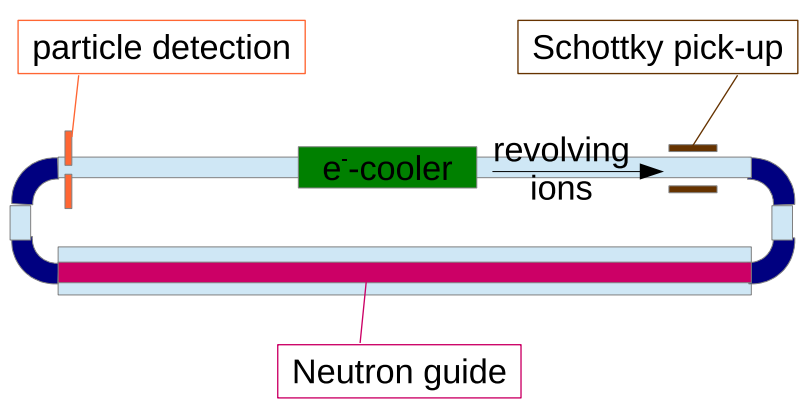

Figure 4. Schematic drawing of the proposed setup. Shown are the main components of an ion storage ring which include the beam lines and focusing elements (blue), dipoles (dark blue), electron cooler (green), a neutron guide parallel to a part of the storage ring (purple), particle detection capability (orange), and Schottky pickup electrodes (brown). The ions are kept at the kinetic energy of interest by employing electron cooling in one of the straight sections of the long ring. The other straight section is the neutron guide. Neutron-induced reaction products have a different magnetic rigidity and thus travel on a separated orbit, which can be detected.

the $(n, \gamma)$ cross sections in reactors doesn't constrain the astrophysical rates significantly. However, since typically the direct capture component can be determined, it can help to improve the extrapolation of measurements in the keV-regime to temperatures not investigated so far. An example for such a measurement is the radioactive isotope ${ }^{60} \mathrm{Fe}[9]$.

A direct usage of reactor neutrons in the astrophysical energy regime is only possible, if the corresponding ions are not at rest in the laboratory frame. Ions with energies between $0.1 \mathrm{AMeV}$ and $10 \mathrm{AMeV}$ can be efficiently stored in ion storage rings $[10,11]$. The combination of such a ring with a reactor could open the path to direct measurements of neutron-induced cross sections on short-lived radioactive isotopes in the keV-regime [12]. The interaction zone could be the reactor core as discussed in [13].

An important alternative could be long neutron guides, see Fig. 4. The advantage compared to the penetration of the reactor core are obviously safety considerations. A second advantage would be the fact that the high-energy neutrons and $\gamma$-rays, which are very abundant close to the reactor core, are suppressed by several orders of magnitude compared to the low-energy neutrons. The reason for this drastic enhancement in (sub) thermal neutrons is the fact that low-energy neutrons are basically trapped inside the neutron guide while the other particle penetrate the walls. The radioactive ions under investigation can then be stored in a long storage ring. One half of the storage ring could then be a neutron guide. The center-of-mass energy is determined by the energy of the revolving ions.

However, the huge disadvantage as compared to a design where the ring passes by the reactor core is the reduced neutron density. In order to stay trapped inside the neutron guide, the neutrons must have a very small angle with respect to the walls. The necessary total reflection is only possible under these conditions. Therefore most of the neutrons entering such a guide will leave it at the next wall - just like the high-energy neutrons and $\gamma$-rays. Typically, the low-energy neutron fluxes inside the guide is $4-5$ orders of magnitude below the fluxes closer to the 
core. This can be partly compensated by the possibility of increasing the length of the interaction zone. The reasonable assumption of a neutron flux of $210^{10} \mathrm{n} / \mathrm{cm}^{2} / \mathrm{s}$ in the neutron guide as available at the research reactor at the ILL, Grenoble, and an interaction zone of $100 \mathrm{~m}$ leads to a neutron target with a density of $10^{9} \mathrm{n} / \mathrm{cm}^{2}$. The luminosity assuming $10^{13}$ ions/s passing the neutron target would then correspond to about 1 count/day/mb.

Such a facility would allow a direct measurement of neutron induced reactions over a wide energy range on isotopes with half lives down to minutes.

This research has received funding from the European Research Council under the European Unions's Seventh Framework Programme (FP/2007-2013) / ERC Grant Agreement No. 615126 and HIC for FAIR.

\section{References}

[1] A. Koloczek, B. Thomas, J. Glorius, R. Plag, M. Pignatari, R. Reifarth, C. Ritter, S. Schmidt, K. Sonnabend, Atomic Data and Nuclear Data Tables 108, 1 (2016)

[2] F.K. Thielemann, C. Fröhlich, R. Hirschi, M. Liebendörfer, I. Dillmann, D. Mocelj, T. Rauscher, G. Martinez-Pinedo, K. Langanke, K. Farouqi et al., Progress in Particle and Nuclear Physics 59, 74 (2007)

[3] R. Reifarth, C. Lederer, F. Käppeler, Journal of Physics G Nuclear Physics 41, 053101 (2014)
[4] F. Herwig, M. Pignatari, P.R. Woodward, D.H. Porter, G. Rockefeller, C.L. Fryer, M. Bennett, R. Hirschi, Ap. J. 727, 89 (2011)

[5] D.A. García-Hernández, O. Zamora, A. Yagüe, S. Uttenthaler, A.I. Karakas, M. Lugaro, P. Ventura, D.L. Lambert, A\&A. 555, L3 (2013)

[6] M. Pignatari, K. Göbel, R. Reifarth, C. Travaglio, International Journal of Modern Physics E 25, 1630003 (2016)

[7] S. Katcoff, O.A. Schaeffer, J.M. Hastings, Phys. Rev. 82, 688 (1951)

[8] M.B. Chadwick, M. Herman, P. Obložinský, M.E. Dunn, Y. Danon, A.C. Kahler, D.L. Smith, B. Pritychenko, G. Arbanas, R. Arcilla et al., Nuclear Data Sheets 112, 2887 (2011)

[9] T. Heftrich, M. Bichler, R. Dressler, K. Eberhardt, A. Endres, J. Glorius, K. Göbel, G. Hampel, M. Heftrich, F. Käppeler et al., Phys. Rev. C 92, 015806 (2015)

[10] M. Lestinsky, V. Andrianov, B. Aurand, V. Bagnoud, D. Bernhardt, H. Beyer, S. Bishop, K. Blaum, A. Bleile, A. Borovik et al., The European Physical Journal Special Topics 225, 797 (2016)

[11] Y.A. Litvinov, S. Bishop, K. Blaum, F. Bosch, C. Brandau, L.X. Chen, I. Dillmann, P. Egelhof, H. Geissel, R.E. Grisenti et al., Nuclear Instruments and Methods in Physics Research B 317, 603 (2013)

[12] J. Glorius, Y.A. Litvinov, R. Reifarth, Physica Scripta Volume T 166, 14008 (2015)

[13] R. Reifarth, Y.A. Litvinov, Phys. Rev. ST Accel. Beams 17, 014701 (2014) 